\title{
Right-half plane zeros are not necessary for inverse response
}

\author{
J.M. Maciejowski
}

\begin{abstract}
The presence of 'inverse response' in the step response of a linear time-invariant system is closely associated with the presence of right-half plane zeros. Various links between the two are known: real positive zeros are sufficient to produce inverse response, and an odd number of positive real zeros is necessary and sufficient to give a particular form of inverse response. On the other hand, the presence of complex right-half plane zeros can result in inverse response, or not. The remaining question is whether the presence of right-half plane zeros is necessary for the presence of inverse response. This paper shows that it is not. This is demonstrated by the generation of a number of counter-examples, using an optimisation algorithm and a particular parametrisation of Hurwitz polynomials. We show that the result holds for a strengthened form of inverse response, which we call ' $\rho$-inverse response', and if the system poles are constrained to be real.
\end{abstract}

\section{INTRODUCTION}

It is commonly believed that 'inverse response' of a linear time-invariant system can arise only from transfer-function zeros with positive real parts. Some specific results are available in the literature:

- A real positive zero is a sufficient condition for inverse response to be exhibited [7].

- An odd number of zeros with positive real part results in the initial slope of a step response being in the "wrong" direction [6], [1], [9].

- Only complex (ie non-real) zeros with positive real parts are not sufficient to cause inverse response (see section II).

What appears to be not known - although it is generally believed - is whether the existence of at least one zero with positive real part is a necessary condition for inverse response to exist. This paper shows, by a number of counter-examples, that right-half plane zeros are not necessary for a system to exhibit inverse response.

We will consider single-input, single-output, stable, continuous-time LTI systems with strictly proper rational transfer functions. Without loss of generality (for our purposes) we shall assume that the transfer function has the form

$$
G(s)=\frac{\left(s-z_{1}\right)\left(s-z_{2}\right) \cdots\left(s-z_{m}\right)}{\left(s-p_{1}\right)\left(s-p_{2}\right) \cdots\left(s-p_{n}\right)}
$$

with $m<n, \Re\left(p_{i}\right)<0$ for $i=1,2, \ldots, n$, and of course any complex zeros and poles occur in conjugate pairs. We

The author is with the Department of Engineering, University of Cambridge, Cambridge CB2 1PZ, UK, and with the Cambridge Centre for Advanced Research and Education (CARES) Ltd, Singapore. jmmeeng.cam.ac.uk

This work was supported by the Singapore National Research Foundation (NRF) under its Campus for Research Excellence and Technological Enterprise (CREATE) programme, specifically the C4T project within CARES. denote the step response of the system - namely the output resulting from a unit step applied at the input at time $t=0$ to the system initially completely at rest - as $y_{s}(t)$. Note that the initial and final values of the step response are given by

$$
y_{s}(0)=0 \quad \text { and } \quad y_{s}(\infty)=\frac{\left(-z_{1}\right)\left(-z_{2}\right) \cdots\left(-z_{m}\right)}{\left(-p_{1}\right)\left(-p_{2}\right) \cdots\left(-p_{n}\right)} .
$$

We shall assume that $z_{i} \neq 0$ for $i=1,2, \ldots, m$ so that $y_{s}(\infty) \neq 0$. Note that, since the system is stable, $y_{s}(\infty)>0$ if the number of zeros with positive real parts is even, and $y_{s}(\infty)<0$ if it is odd.

We shall say that the system exhibits inverse response if there is an interval $\left[t_{1}, t_{2}\right]$ such that

$$
\operatorname{sign} y_{s}(t)=-\operatorname{sign} y_{s}(\infty) \text { for } t \in\left[t_{1}, t_{2}\right]
$$

Figure 1 shows the step responses of the systems

$$
G_{1}(s)=\frac{1-s}{(1+s)^{3}}, \quad G_{2}(s)=\frac{(1-s)^{2}}{(1+s)^{3}}
$$

respectively, both of which exhibit inverse response in this sense. Systems which exhibit inverse response occur in

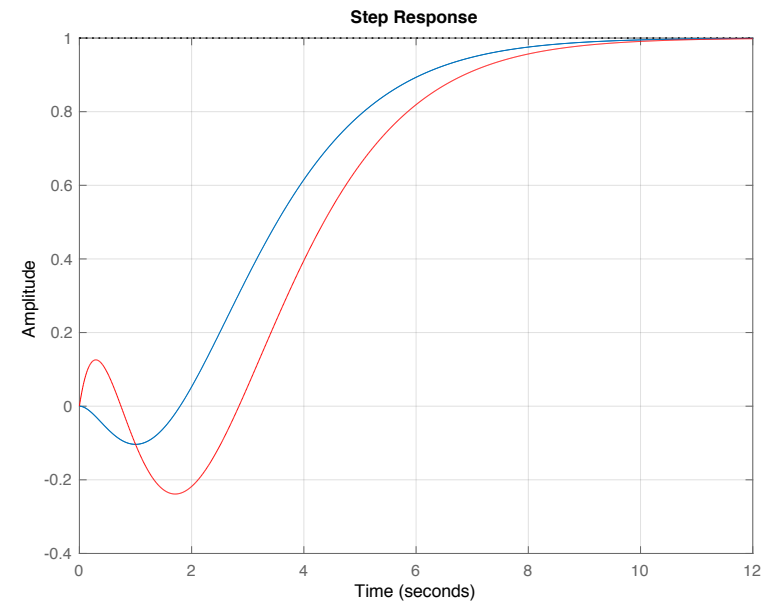

Fig. 1: Step responses of $G_{1}(s)$ (solid, blue) and $G_{2}(s)$ (dashed, red).

aerospace applications (eg elevator to altitude response of an aircraft), process control (eg water feedrate to boiler water level), neuroscience (eg the classical Hodgkin-Huxley 'voltage clamp' experiment) and economics (eg exchange rate to balance of payments response).

Note that some authors use the term undershoot instead of 'inverse response' [7], [9]. We prefer not to use this 
term, because in process control its meaning is somewhat different: it often refers to a response which almost reaches its final value, then 'pauses' or 'backs off' before continuing to its final value (possibly also overshooting the final value). Figure 2 illustrates the difference between this meaning of 'undershoot' and 'inverse response': the red curve is the step response of the system

$$
\frac{0.3 s^{2}+7 s+1}{10 s^{3}+8 s^{2}+11 s+1}
$$

and displays 'undershoot', while the blue curve is the step response of the system

$$
\frac{0.5 s^{2}-s+1}{(s+1)^{3}}
$$

which displays 'inverse response'. (Several other terms and meanings can be found, for example 'undershoot' meaning overshoot when the set-point is negative, or the negative swing of an oscillatory response, or 'pre-transition undershoot' for what we call 'inverse response'.)

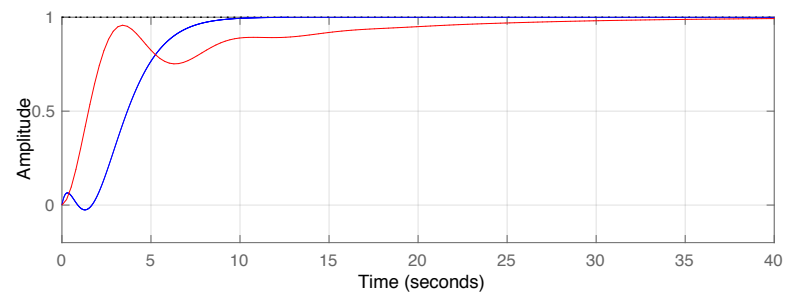

Fig. 2: Illustrating terminology: The red curve exhibits 'undershoot', while the blue curve exhibits 'inverse response'.

\section{WHAT IS ALREADY KNOWN}

Assume that the tranfser function is in the form (1). From the Initial Value Theorem for Laplace transforms it follows immediately that the first $n-m-1$ derivatives of $y_{s}(t)$ are zero at $t=0$, and the first non-zero derivative is

$$
\left.\frac{d^{n-m} y_{s}(t)}{d t^{n-m}}\right|_{t=0}=1
$$

Thus $y_{s}(t)>0$ for sufficiently small $t$, and, from (2), inverse response is certainly exhibited if there is an odd number of $z_{i}$ 's with positive real values [6], [1], [9] — which is equivalent to there being an odd number of zeros with positive real parts.

A more general sufficient condition for inverse response is known:

Theorem [5], [7]: If $G(z)=0$ for a positive real value of $z$ then the system exhibits inverse response.

Proof [5], [7]: Let $Y_{s}(s)$ denote the Laplace transform of $y_{s}(t)$. If $G(z)=0$ then $Y_{s}(z)=G(z) / z=0$. Hence

$$
0=Y_{s}(z)=\int_{0}^{\infty} y_{s}(t) e^{-z t} d t
$$

But $e^{-z t}>0$ so $y_{s}(t)$ must take both positive and negative values.
Note that this argument fails for negative real zeros, because they are outside the region of convergence of the integral, due to $y_{s}(\infty)$ being non-zero.

But if all the right-half plane zeros have non-zero imaginary parts then inverse response may not be exhibited, so the presence of right-half plane zeros is not a sufficient condition for inverse response. This is shown by the counter-example

$$
G(s)=\frac{s^{2}-0.2 s+2}{(s+1)^{3}}
$$

which has zeros at $0.1 \pm 1.4107 i$. On the other hand, inverse response may occur with only complex right-half plane zeros, as shown by the example

$$
G(s)=\frac{s^{2}-2 s+2}{(s+1)^{3}}
$$

which has zeros at $1 \pm i$. Figure 3 shows the step responses of these two systems.

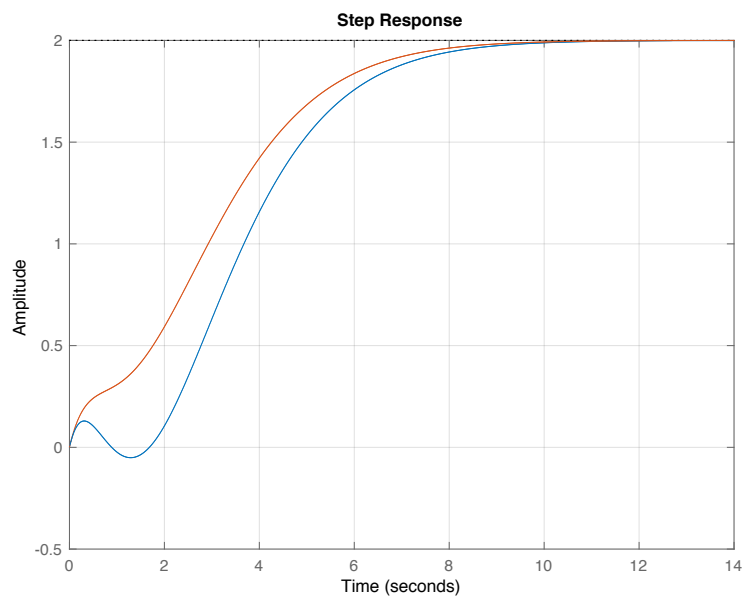

Fig. 3: Step responses of the systems (9) (with no inverse response) and (10) (with clear inverse response).

The remaining question is whether the presence of righthalf plane zeros is necessary for inverse response to occur in the general case, namely when complex poles and/or zeros are allowed, or when all the poles and zeros are real but the number of zero-crossings by the step response is even. We shall provide a number of counter-examples showing that such zeros are not necessary for inverse response.

\section{RHP ZEROS ARE NOT NECESSARY FOR INVERSE RESPONSE}

\section{A. Counter-examples and a modified definition}

Right half-plane zeros are not necessary to obtain inverse response as defined by (3). A counter-example is the following system:

$$
\frac{s+0.08707}{s^{2}+6.041 s+101.4}
$$

which has a zero at -0.0871 , poles at $-3.02 \pm 9.60 i, y_{s}^{\prime}(0)>$ $0, y_{s}(\infty)=8.6 \times 10^{-4}>0$, and crosses 0 several times. Its step response is shown in Fig.4. Its DC gain is almost 0, in comparison with its peak value. 


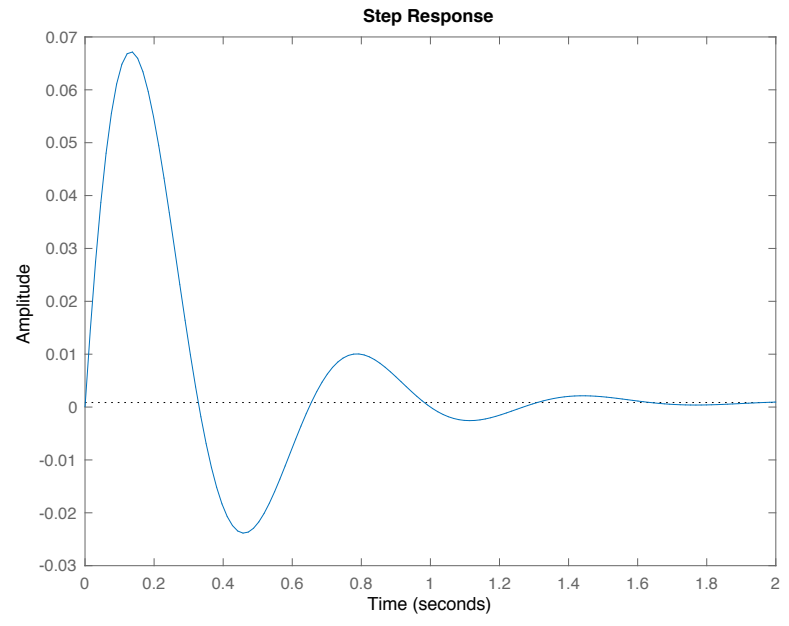

Fig. 4: Step response of the system (11).

Most control engineers would probably object that a response such as the one shown in Fig.4 is not what they mean by 'inverse response', because its final value is effectively zero. We therefore introduce the following modified definition.

Definition Let the peak value of the step-response $y_{s}(t)$ be

$$
\bar{y}_{s}=\sup _{t>0}\left|y_{s}(t)\right|
$$

Then the step response exhibits $\rho$-inverse response if $y_{s}\left(t_{0}\right)=0$ for some $t_{0}>0$ and

$$
\frac{\left|y_{s}(\infty)\right|}{\bar{y}_{s}}=\rho .
$$

Clearly $0<\rho \leq 1$ according to this definition (recalling that $z_{i} \neq 0$ by assumption). Our contention is that what is usually meant by the term "inverse response" is " $\rho$-inverse response with $\rho$ 'close to' 1". The system (11) exhibits "0.013-inverse response", whereas (10) exhibits "1-inverse response".

The third-order system

$$
\frac{1.953 s^{2}+1.157 s+0.8897}{0.3553 s^{3}+0.8811 s^{2}+6.508 s+0.2559}
$$

has poles at -0.0395 and $-1.2200 \pm 4.0901 i$ and zeros at $-0.2964 \pm 0.6064 i$. It exhibits $\rho$-inverse response with $\rho=1$. The most negative value of its step response is $y_{s}(1.13)=$ -0.019 , and its final value is $y_{s}(\infty)=3.48$. A portion of its step response is shown in Fig.5. This example demonstrates that RHP zeros are not necessary for 1-inverse response.

\section{B. Systems with real poles only}

The counter-example above has complex poles, and relies on the negative swings of very underdamped modes.This again is not what is typically meant by 'inverse response'. We shall therefore limit attention now to systems with real poles only.

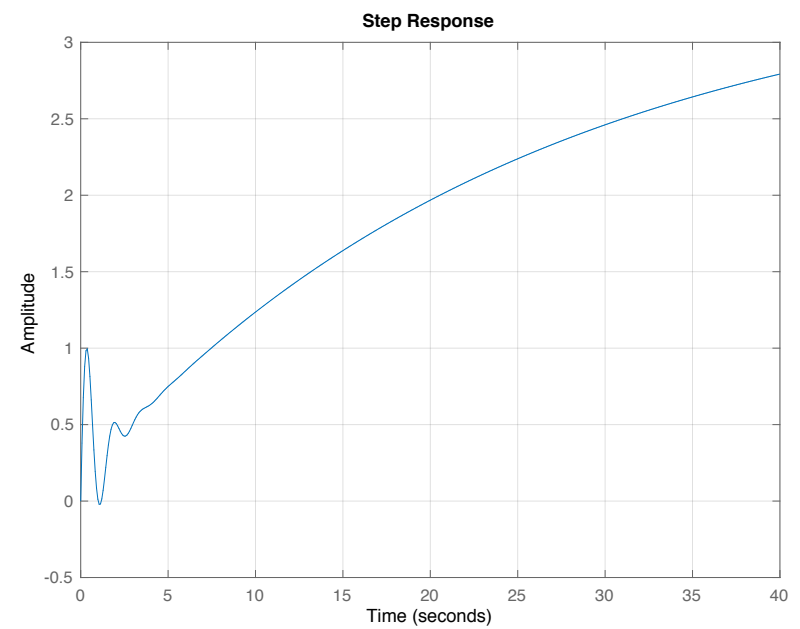

Fig. 5: Step response of the system (14).

The fourth-order system

$$
\frac{7.282 s^{3}+0.3043 s^{2}+0.4029 s+0.01099}{0.1107 s^{4}+1.411 s^{3}+5.992 s^{2}+8.483 s+0.003064}
$$

has poles at $-4.3453,-4.2058,-4.1918,-0.0004$ and zeros at $-0.0072 \pm 0.2343 i$ and -0.0275 . It exhibits $\rho$-inverse response with $\rho=1$. Its step response is shown in Fig.6 on two very different time scales.

Note that such clear time-scale separation is not necessary to achieve 1-inverse response without RHP zeros. For example, for the following 6th-order system, the ratio of the fastest to the slowest pole is $2.2226 / 0.0279 \approx 80$.

$$
\begin{gathered}
7.688 s^{5}+6.043 s^{4}+9.882 s^{3}+5.594 s^{2} \\
+2.088 s+0.7047 \\
\hline 0.1896 s^{6}+2.078 s^{5}+9.12 s^{4}+20.07 s^{3}+22.21 s^{2} \\
+10.07 s+0.2639
\end{gathered}
$$

This has poles at $-2.2226,-2.2142,-2.1866,-2.1750$, $-2.1343,-0.0279$ and zeros at $-0.0710 \pm 0.9521 i$, $-0.0780 \pm 0.4472 i$ and -0.4879 . The step response of this system is shown in Fig.7, and it is seen that it exhibits 1inverse response.

For the following 9th-order system the ratio of the fastest to the slowest pole is $2.443 / 0.1224 \approx 20$ :

$$
\begin{gathered}
0.2893 s^{8}+18.76 s^{7}+27.2 s^{6}+59.8 s^{5}+63.03 s^{4} \\
+44.33 s^{3}+32.49 s^{2}+6.723 s+3.395 \\
\hline 0.08863 s^{9}+1.386 s^{8}+9.454 s^{7}+36.73 s^{6}+89.04 s^{5} \\
+138.3 s^{4}+135.3 s^{3}+77.85 s^{2}+22.16 s+1.765
\end{gathered}
$$

Again, this system has all its zeros in the open left-half plane. Its step response is shown in Fig.8.

A striking feature of all the systems (with real poles) that we have generated to date, and that reach substantially negative values, is that their step response has an initial fast transient which reaches the ultimate final value, then returns to near zero, before eventually (and relatively slowly) increasing to the final value. It seems unlikely that this is a 


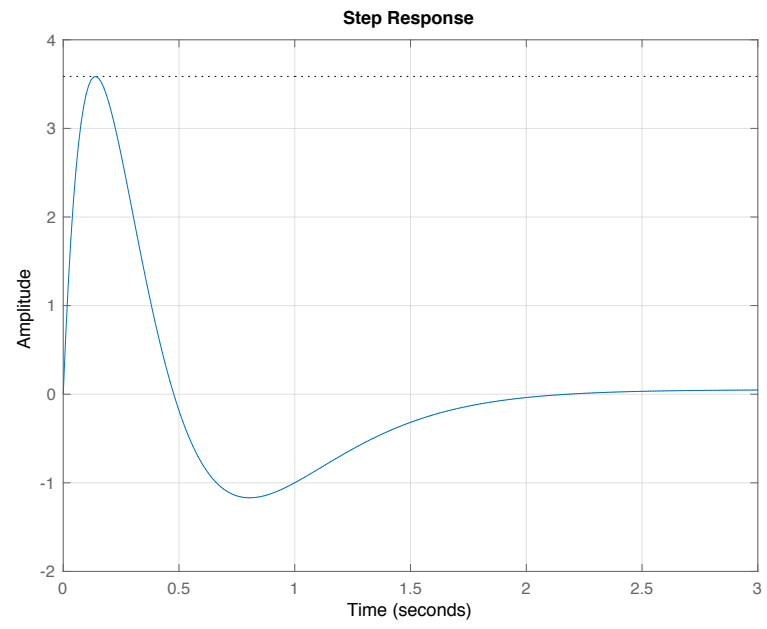

(a) Step response over $0<t<3$.

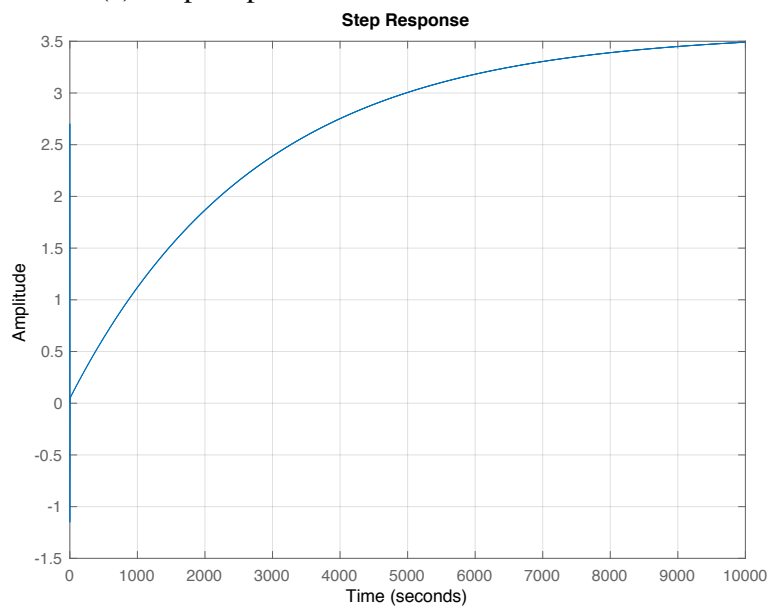

(b) Step response over $0<t<10^{4}$.

Fig. 6: Step response of (15) over two different time scales. The dotted line shows the final value.

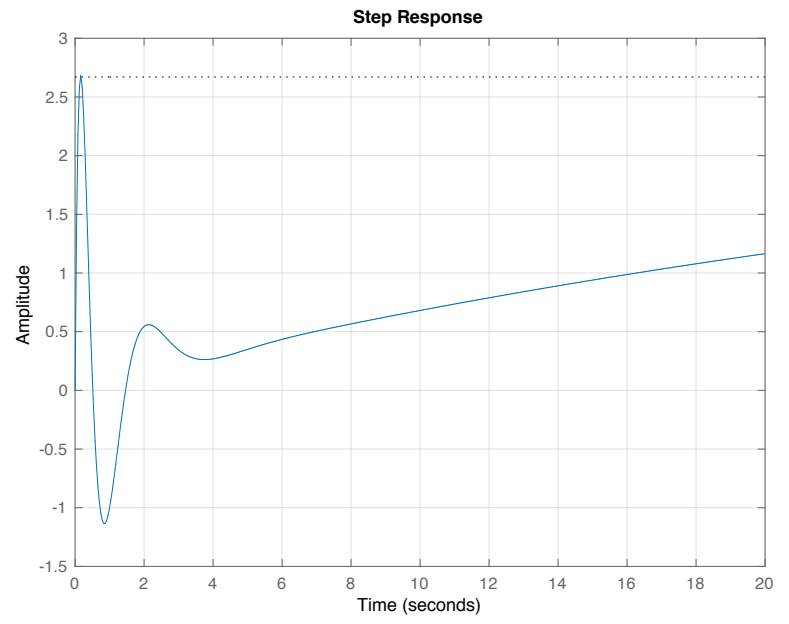

Fig. 7: Step response of the system (16).

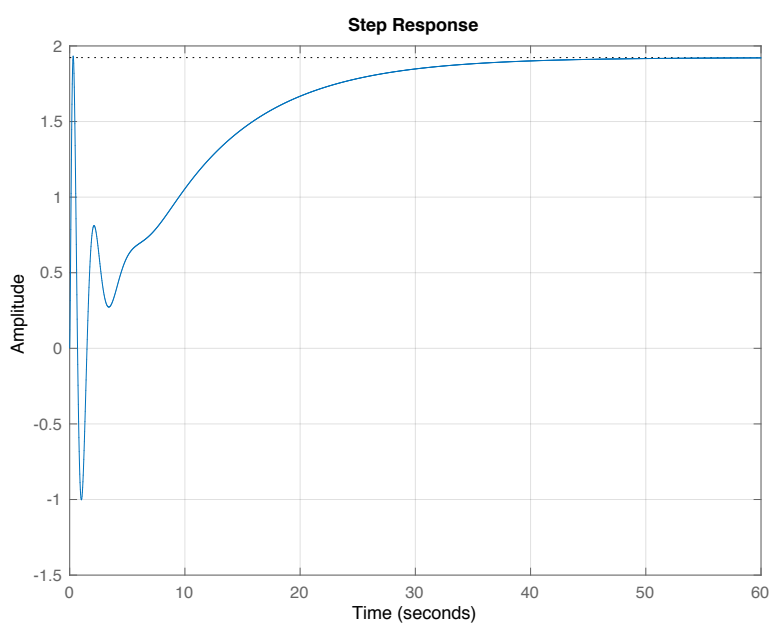

Fig. 8: Step response of the system (17).

necessary feature, but at the time of writing this has not been investigated further.

\section{IMPLICATIONS FOR FEEDBACK PERFORMANCE}

Intuitively, inverse response impedes the achievable control performance of a system. This intuition is made precise by the well-known fact that right-half plane zeros limit the achievable bandwidth of feedback systems [3]. Roughly speaking, an upper bound on the achievable closed-loop bandwidth is given by the modulus of the right-half plane zero nearest to 0. (See [5] for a more accurate estimate.)

Systems (15) and (16) have zeros very close to the imaginary axis, at $-0.0072 \pm 0.2343 i$ and $-0.0710 \pm 0.9521 i$, respectively. These can be expected to impose a bandwidth limitation in practice, since a practical design must have some robustness to model errors and plant variations which may result in these zeros crossing over the axis into the righthalf plane.

But this is not the case for system (18):

$$
\begin{aligned}
& 0.03904 s^{8}+32990 s^{7}+12550 s^{6}+41920 e 04 s^{5} \\
& \quad+12210 s^{4}+16030 s^{3}+2620 s^{2}+1801 s+1.192 \\
& \hline s^{9}+432.6 s^{8}+4963 s^{7}+23350 s^{6}+57250 s^{5} \\
& \quad+77500 s^{4}+56060 s^{3}+18790 s^{2}+1905 s+0.2631
\end{aligned}
$$

It has real poles and exhibits 1-inverse response, as shown in Fig.9 (on two time scales), but its zeros are located at $-8.4498 \times 10^{5},-6.6269 \times 10^{4},-1.5655 \times 10^{3} \pm$ $81.032 i,-478.39 \pm 58.595 i,-14.043 \pm 46.966 i$. The greatest angle that any of these make with the negative real axis is $\arctan (47 / 14)=73$ degrees. Thus all the zeros are likely to remain in the left-half plane even with considerable plant variations, and therefore do not appear to impose any inherent bandwidth limitations on achievable feedback designs. This is presumably due to the very fast initial transient of the step response in the "correct" direction. These points remain to be investigated more thoroughly. 


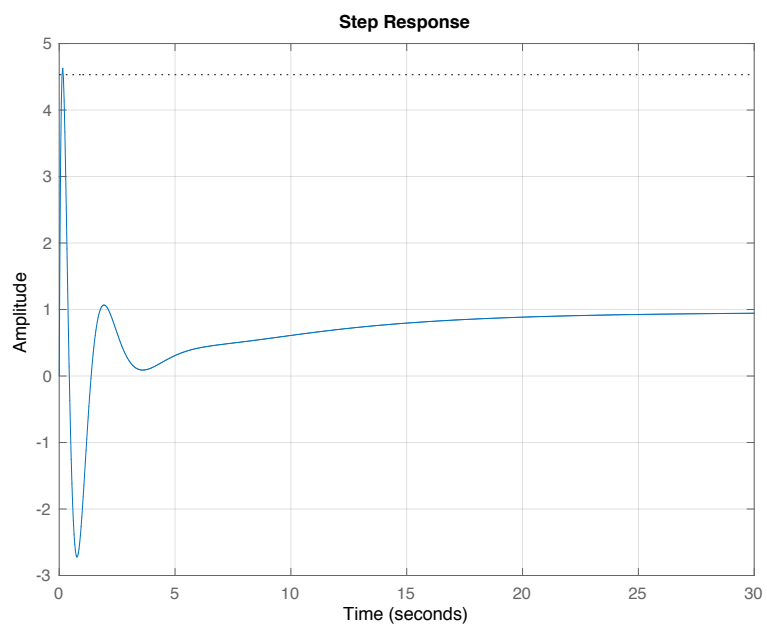

(a) Step response over $0<t<30$.

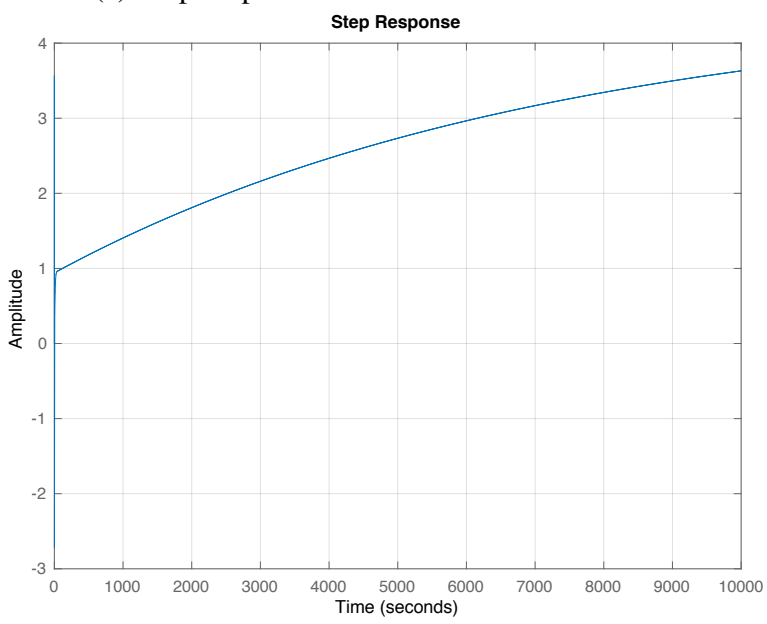

(b) Step response over $0<t<10^{4}$.

Fig. 9: Step response of (18) over two different time scales. The dotted line shows the final value.

\section{Generation of COUNTER-EXAMPLES}

The counter-examples given in section III were generated by searching, using an optimisation algorithm. To make the search more efficient and well-behaved we search over a bounded domain, the obvious one being the interior of the unit disk. We use the Levinson-Durbin parametrisation of Schur-stable polynomials [8, Lemma 16.1, p.240], together with a standard bilinear mapping [2]. When searching over systems with real poles only, we parametrise Schur polynomials by their (real) roots; these are mapped to real roots of Hurwitz-stable polynomials by the bilinear mapping.

We search by minimising $\left|y_{s}(1)-\alpha\right|$ over specific sets of systems, with $\alpha=0$ or $\alpha=-1$. Note that specifying the value of the step response at $t=1$ does not lose generality, because of the time-scaling theorem of the Laplace transform: $f(t / \beta) \leftrightarrow \beta F(\beta s)$. Thus scaling by any $\beta>0$ does not change conclusions about right-half plane zeros.

Constraints such as achieving a minimum value of $\rho$ (defined in section III-A) are included in the optimisation problem. The search algorithm that we have used is Matlab's fmincon with default options, namely using an interior point algorithm. We have found this to be sufficient, despite having a non-convex optimisation problem. Note that our problem is relatively easy, as we do not need to ensure that we have found a global optimum; we can stop the search as soon as a counter-example has been found. In each case we have specified the relative degree of the systems being searched over to be 1 , since the search algorithm is capable of selecting larger relative degrees, in effect, by selecting zeros with arbitrarily large negative real parts (as happens in the case of system (18), for example).

Our experience is that the search among high-order systems is significantly faster than among low-order systems. It seems that, despite the higher dimension of the search space, it is easier for the search algorithm to find a solution, presumably because of the increased degrees of freedom that are available.

\section{CONCLUSION}

This paper has shown that right-half plane zeros are not necessary to give inverse response, as defined by (3). This remains true even if the concept of inverse response is refined to ' $\rho$-inverse response', with $\rho=1$, (see section III-A), and if poles are constrained to be real. However, systems which have left-half plane zeros only and which exhibit inverse response tend to have rather complicated behaviours, as shown in the various examples presented in sections III and IV.

If one is building a 'black-box' model which exhibits inverse response, then it is probably a good idea to allow it to have right-half plane zeros - for convenience and possibly for credibility of the resulting response. But one is not justified in insisting that an inverse response behaviour must imply the presence of right-half plane zeros.

Several aspects of inverse response behaviour remain to be investigated; in particular its implications for achievable feedback performance. A similar investigation should also be performed for discrete-time systems, for which some initial results are available in [4].

\section{REFERENCES}

[1] R.N. Clark. Introduction to Automatic Control Systems. Wiley, New York, 1962.

[2] D. Hertz. On fast multivariable bilinear and Hadamard transforms. IEEE Transactions on Signal Processing, 39(8):1788 - 1792, August 1991.

[3] I. Horowitz. Synthesis of Feedback Systems. Academic Press, 1963.

[4] L.H. McWilliams and M.K. Sain. Qualitative features of discrete-time system responses. In Proc. 33rd Conference on Decision and Control, Lake Buena Vista, FL, December 1994. IEEE.

[5] R.H. Middleton. Trade-offs in linear control system design. Automatica, 27(2):281-292, February 1991.

[6] T. Norimatsu and M. Ito. On the zero non-regular feedback control systems (in Japanese). Automatic Control, 8(2):78-86, 1961.

[7] J. Stewart and D.E. Davison. On overshoot and nonminimum phase zeros. IEEE Transactions on Automatic Control, 51(8):1378 - 1382, August 2006.

[8] R. Tempo, G. Calafiore, and F. Dabbene. Randomized Algorithms for Analysis and Control of Uncertain Systems. Springer Verlag, 2012.

[9] M. Vidyasagar. On undershoot and nonminimum phase zeros. IEEE Transactions on Automatic Control, AC-31(5):440, May 1986. 